\title{
Structure activity relations of aromatic amino acid derivatives to interact with organic anion transporter OAT1 reveal critical moieties for renal accumulation of tumor imaging probes
}

\author{
Chunhuan Jin ${ }^{1}$, Ryuichi Ohgaki ${ }^{1}$, Hideyuki Tominaga ${ }^{2}$, Suguru Okuda ${ }^{1}$, Shushi Nagamori ${ }^{1,3}$, \\ Yoshikatsu Kanai ${ }^{1}$ \\ ${ }^{I}$ Graduate School of Medicine, Osaka University, Bio-system Pharmacology, Japan, ${ }^{2}$ Advanced Clinical Research \\ Center, Fukushima Medical University, Fukushima, Japan, ${ }^{3}$ Laboratory of Biomolecular Dynamics, Department of \\ Collaborative Research, Nara Medical University, Nara, Japan
}

3-[18F]fluoro-alpha-methyl-L-tyrosine ([18F]FAMT) and 3-[123I]iodo-alpha-methyl-L-tyrosine ([123I]IMT) have been developed as amino acid probes for tumor imaging. FAMT and IMT are accumulated specifically in tumor due to their high selectivity to amino acid transporter LAT1 (L-type amino acid transporter 1) expressed specifically in tumor cells. FAMT and IMT, however, show a high degree of accumulation only in the kidney, which generates strong physiological backgrounds. The renal accumulation of IMT is suppressed by probenecid, suggesting a contribution of organic anion transporters. Our previous study confirmed that FAMT is a substrate of organic ion transporters. Among them, OAT1 that mediates urinary excretion of organic anions is supposed to be the most important because the renal excretion of the probe was inhibited by probenecid. Therefore, we speculate that OAT1 plays a pivotal role in the accumulation of FAMT and IMT in the kidney.

Method: We used a series of aromatic amino acid derivatives with the altered positions for hydroxyl groups and halogen groups on the aromatic rings. The effects of this series of compounds on the OAT1 were examined by uptake experiment and efflux experiment. We investigated whether these compounds interact with OAT1 in order to determine the key structure in FAMT and IMT to be taken up by OAT1.

Result: By comparing IC50s of FAMT, IMT and a series of aromatic amino acid derivatives to inhibit the uptake of paraaminohippurate by OAT1, and their efflux profiles in the efflux experiment, we revealed that both halogen and hydroxyl group on the benzene ring of FAMT and IMT are critical for the interaction, whereas the alpha-methyl moiety essential for the selectivity to LAT1 is not important to interact with OAT1. We also found that the position of hydroxyl group and halogen group on the benzene ring affects the interaction with OAT1, suggesting the importance of hydrophobicity around particular position of the aromatic ring to form a hydrophobic core, which is essential to be the substrates of OATmember transporters.

Conclusion: The results of this experiment would benefit the design of the tumor-specific imaging probes with less renal background. 